\title{
EL PROGRAMA DE CAPACITACIÓN EMPRESARIAL SE RELACIONA CON EL DESEMPEÑO LABORAL CASO: TRABAJADORES DEL FONDO DE EMPLEADOS DEL BANCO DE LA NACIÓN
}

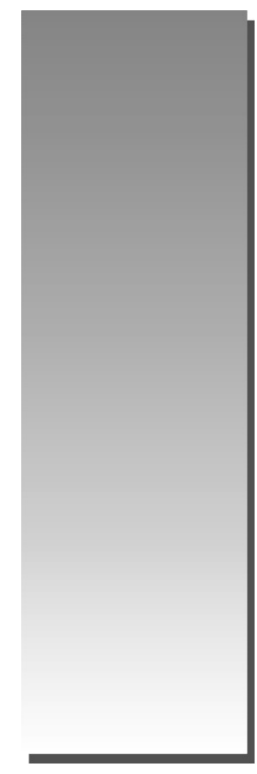

Dr. Jorge V. Mayurí Barrón *

jvmayuri@hotmail.com

\section{RESUMEN}

Hoy existe una alta presión competitiva, esto hace que la empresas tengan que alinearse con su respectiva competencia y más aun con los deseos de los clientes, razón por la cual se crean las empresas; muchas empresas consideran que para estar actualizadas en los avances competitivos deben de considerar solamente el mantenimiento de sus diferentes recursos como materiales, equipos, entre otros. Muchas de ellas se olvidan que el potencial humano requiere su respectiva actualización, por ello la creación de programas de capacitación empresarial acorde a lo que el mercado requiere es de suma importancia, ya que ello permitirá incrementar el desempeño laboral y, por ende, la productividad laboral.

Palabras claves: Presión Competitiva, Clientes, Potencial Humano, Programas, capacitación, Mercado, Productividad.

\section{ABSTRACT}

Today there is a high competitive pressure, this makes the companies have to align with their competence and even more with the wishes of customers, which is why companies are created, many companies find that to be updated on the progress competitive must consider only the maintenance of its various resources such as materials, equipment and others, many of them forget that human potential requires their respective update, thus the creation of corporate training programs in line with what the market is required important as this will enhance job performance and hence labor productivity.

Keywords: Competitive Pressure, Clients, Human Potential, Programs, Training, Market, Productivity.

\footnotetext{
* Doctor en Educación (UNMSM), Magister en Administración, con Mención en Gestión Empresarial (UNMSM); Licenciado en Administración de Empresas (Universidad Ricardo Palma) Post Grado en Derecho y Espacial (UNMSM), Profesor Asociado de la Facultad de Ciencias Administrativas de la UNMSM, Profesor de Post Grado en: USMP, UNFV, UNSCH, UNCP, ESAN, Capacitador Empresarial.
} 


\section{INTRODUCCIÓN}

Las organizaciones empresariales, sean empresas o instituciones sin fines de lucro, constituyen la fuente que impulsa el desarrollo de las colectividades y, por ende, del conjunto de todas ellas que constituyen la sociedad.

Una sociedad es calificada de buena o mala dependiendo del nivel de desarrollo alcanzado por sus colectividades, que se mide por su nivel de estructuración de los valores que se predican y los que se practican, la normatividad, los protocolos, las costumbres en suma, por su cultura organizacional, la cual se expresa en el desempeño laboral de los integrantes del sistema en sus conjunto.

Cuando el sistema falla, es necesario replantear la existencia misma de la organización y de cada uno de sus componentes, comparando el rendimiento idealizado con el real y estableciendo las causas de las "fracturas o distorsiones", con la finalidad de proponer correcciones y establecer un nuevo rumbo acorde a las necesidades actuales y proyectadas de la organización.

La corrección se introduce mediante un programa de capacitación que permita cubrir los vacíos identificables en el diagnóstico y con ello iniciar el proceso de transformación, en el núcleo de la cultura organizacional, a fin de que los cambios introducidos se consoliden y, por ende, se eleve el rendimiento del sistema empresarial.

Esto implica que la realización de un estudio, se ubique en el campo educativo al pretender transformar las costumbres, en este caso del personal que labora en el Fondo de Empleados del Banco de la Nación - FEBAN.

El Fondo de Empleados del Banco de la Nación, conocido por sus siglas FEBAN, es una institución de los empleados del Banco de la Nación con personería jurídica de derecho privado, que tiene por finalidad proporcionar a los trabajadores y ex trabajadores de la mencionada cantidad y sus familiares, los beneficios y servicios de asistencia social, salud, vivienda, préstamos, fondo de seguro de vida y otros que apruebe la Comisión de Administración del Fondo de Empleados.

El presente estudio detalla la aproximación al problema a investigar, los fines que se pretenden cumplir, las hipótesis sostenidas y la relación de variables involucradas que son desarrolladas en los cuadros de operacionalización correspondientes, presenta los instrumentos a emplear para su culminación.

\section{MÉTODOS}

\section{DESCRIPCIÓN DEL PROBLEMA}

La manera en que interpretamos la realidad, realizamos las tareas encomendadas y con ellos movilizamos socialmente a la estructura organizacional llamada empresa o institución, está premunida de valores y a todo este conglomerado se le denomina Cultura Organizacional.

La cultura organizacional, esta dada por el conjunto de conductas, ritos, creencias, anécdotas, protocolos, procesos y procedimientos que definen el que las instituciones se orienten hacia la excelencia o al fracaso; con el paso del tiempo el conjunto de experiencias empresariales va asumiendo un carácter antrópico (desordenado) requiriendo un esfuerzo de corrección que puede provenir de cualquier teoría de gestión empresarial debiendo ser administrada mediante un programa de capacitación empresarial.

Esto permite que la tesis, eminentemente se desarrolle en el campo educativo ya que es a través de un programa en capacitación empresarial que se pretenden introducir cambios en la cultura para reorientar a la organización hacia la excelencia con la sociedad de la que toma recursos y a la que sirve.

\section{PLANTEAMIENTO DEL PROBLEMA}

\section{ANTECEDENTES}

El origen de la Cultura Organizacional se remonta a la existencia misma del hombre, como animal gregario y a la necesidad de otorgar dirección y garantizar el funcionamiento y supervivencia de las organizaciones que ha ido formando a lo largo de su vida, para que no requiera de la dependencia de una persona, solo del sistema del que forma parte.

A lo largo de la historia de la civilización, las organizaciones han sufrido modificaciones en cuanto a su complejidad y a la variedad de servicios que prestan siendo su funcionamiento respaldado por la existencia de una cultura organizacional cohesionada en torno a los valores de sus líderes.

La evolución histórica de las transformaciones culturales, nos han revelado la presencia de los factores que permiten construir una cultura: 
- Liderazgo,

- Visión de futuro,

- Objetivos y metas,

- Valores, y rituales,

- Símbolos,

- La construcción de un lenguaje propio,

- Los relatos y anécdotas,

- La prospectiva

De estos factores, el debilitamiento de su capacidad de integralidad y el ingreso de la entropía organizacional ha propiciado la caída motivacional que se ha traducido en la existencia de un Problema de Cultura, que se manifiesta en un clima desarticulado que condiciona el desempeño Organizacional.

En el FEBAN, se pueden apreciar distorsiones cuya complejidad y magnitud limitan las posibilidades de gestión de estrategas y decisores ante imprecisiones en la visión de futuro, objetivos y metas debido a una contraposición de liderazgos que encubren problemas de valores y clima organizacional, que dividen a los equipos de trabajo, restringiendo las posibilidades de servicio a los afiliados.

\section{DIAGNÓSTICO DE LA SITUACIÓN ACTUAL}

A nivel del contexto global, se puede apreciar que las organizaciones empresariales de alto desempeño en los países del primer mundo y en los emergentes se caracterizan por una Cultura organizacional diferenciada y que han convertido en ventaja competitiva.

Esta situación apreciada en mega mercados como EEUU y UE, revelan un paradigma educativo de éxito, consistente en el empleo de Programas de Capacitación específicos y cuya calendarización tiene una cobertura Anual.

En América Latina las principales empresas han logrado superar dificultades de contexto que amenazaban su existencia en base a Programas de Capacitación de diversa duración y cuyos resultados se han revelado en la generación de estrategias exitosas, que han revitalizado el sistema de la Cultura Organizacional.

En el Perú, la cultura organizacional como esencia de un sistema empresarial sólido no ha sido lo suficientemente explorado, por privilegiar factores cuantitativos, financieros y de produc- ción, que no reconocen la esencia del factor humano como activador de desarrollo y como ente reprogramable en cuanto a sus capacidades de aprendizaje de novedosas formas de actuación, basados en la falsa premisa del "divide y reinarás" que ha incubado climas hostiles y en los que las iniciativas empresariales se detienen y combaten impidiendo a la empresa avanzar al cumplimiento de sus metas.

La problemática cultural que afecta al desempeño organizacional se traduce en un burocratismo excesivo en el que se pierde de vista la misión y visión empresarial, los objetivos y metas se vuelven confusos, los procesos se distorsionan, la comunicación y las referencias de valor también, aumentando la desmotivación, disminuyendo la productividad, incrementando costos y complejidad de procesos sin agregar valor al cliente, situación que ha venido afectando al Fondo de empleados del Banco de la Nación-FEBAN.

\section{PROYECCIÓN DE LA SITUACIÓN PROBLEMÁTICA}

De persistir las condiciones actuales en el FEBAN, la duplicidad de funciones, procesos, reportes, el debilitamiento de la autoridad, las distorsiones con que se presentan las nuevas situaciones ante las autoridades, las pequeñas "venganzas" que marginan de la gestión a funcionarios calificados por haber pertenecido a una gestión anterior, pueden contribuir a crear una situación de postración y anquilosamiento que perjudicaría los intereses de los usuarios del FEBAN.

\section{CONTROL AL PRONÓSTICO}

Entre el conjunto de medidas asumidas para contrarrestar esta problemática se consideraron:

- Generación de una línea de base que revelase la actual situación con las particularidades propias de la cultura organizacional que actualmente rige al FEBAN;

- Reuniones sociales en la sede institucional con la finalidad de establecer lazos de armonía entre sus integrantes (personal operativo) sin embargo, se pudo apreciar que más allá del episódico momento la situación no cambiaba.

La gerencia recurrió a eventos eclesiásticos con la finalidad de sensibilizar las diversas posiciones, sin embargo el entusiasmo decayó.

Ante esta situación se plantea la presente investigación para establecer, si un programa de capacitación empresarial pudiese representar la 
mejor alternativa para corregir las distorsiones apreciadas a nivel de la cultura organizacional del FEBAN.

\section{FORMULACIÓN DEL PROBLEMA}

¿De qué manera un programa de capacitación empresarial se relaciona con el desempeño laboral de los trabajadores del FEBAN?

\section{PROBLEMAS ESPECIFICOS}

1.- ¿De qué manera un Programa de Capacitación Empresarial dirigido al Grupo A se relaciona con el desempeño laboral del Grupo Control de los trabajadores del FEBAN?

2.- ¿De qué manera un programa de capacitación Empresarial dirigido al Grupo B se relaciona con el desempeño laboral del grupo control de los trabajadores del FEBAN?

3.- ¿De qué manera un Programa de Capacitación Empresarial dirigido al Grupo $\mathrm{C}$ se relaciona con el desempeño laboral del Grupo Control de los trabajadores del FEBAN?

\section{OBJETIVOS DE LA INVESTIGACIÓN}

Han sido identificados los propósitos de la investigación; el general y los específicos, estos últimos derivados de las interrogantes planteadas.

\section{OBJETIVO GENERAL}

Determinar y evaluar de qué manera un Programa de Capacitación Empresarial se relaciona con el desempeño laboral de los trabajadores del Fondo de Empleados del Banco de la Nación.

\section{OBJETIVOS ESPECÍFICOS}

1.- Determinar y evaluar de qué manera un programa de capacitación empresarial dirigido al Grupo A se relaciona con el desempeño laboral del Grupo Control de los trabajadores del FEBAN.

2.- Determinar y Evaluar de que manera un Programa de Capacitación Empresarial dirigido al Grupo B se relaciona con el Desempeño Laboral del Grupo Control de los trabajadores del Fondo de Empleados del Banco de la Nación.

3.- Determinar y evaluar de qué manera un programa de capacitación empresarial dirigido al Grupo C se relaciona con el desempeño Laboral del Grupo Control de los trabajadores del FEBAN.

\section{JUSTIFICACIÓN DE LA INVESTIGACIÓN}

La investigación ha considerado un conjunto de criterios entre los que se tiene:

\section{JUSTIFICACIÓN EMPRESARIAL}

Trascendencia: las Organizaciones empresariales que tienen características similares al FEBAN, pueden implementar un programa de capacitación empresarial similar al que se presenta.

Viabilidad: la realización de la investigación está garantizada al estar vinculado a la empresa por función y como tal garantizar el acceso a la información requerida.

Pertinencia: El momento en que se realizó la investigación fue el más adecuado, ya que de no realizarse habría significado un "punto de ruptura” en la trayectoria de la institución.

Coherencia: La concatenación de los temas ha sido cuidadosamente engarzada desde una perspectiva lógica.

Consistencia: La temática cultural se consolida en base a los resultados trazados (productividad, rentabilidad).

\section{JUSTIFICACIÓN TEÓRICA}

En base a las diversas carencias identificadas en el diagnóstico, se ha construido el programa de capacitación empresarial, que pretende intervenir como variable independiente o solución a corregir el problema identificado en el problema de cultura organizacional que tiene el FEBAN; por esta razón se pretende validar el programa.

\section{JUSTIFICACIÓN PRÁCTICA}

Se pretende resolver un tema de Competitividad, productividad, rentabilidad que dependen directamente de la cultura organizacional, la que, a su vez, depende de dos factores: motivación y conocimiento, los que se expresan en un programa de capacitación empresarial.

\section{JUSTIFICACIÓN METODOLÓGICA}

El estudio emplea a la metodología científica al plantear cuestionamientos, definir propósitos, establecer experimentalmente una prueba para el programa, monitoreando y evaluando sus resultados.

Asimismo, se pretende generar un instrumento de recolección de datos que facilite la información requerida, a partir de la identificación de variables a evaluar. 


\section{HIPÓTESIS DE LA INVESTIGACIÓN}

Las hipótesis planteadas se derivaron del planteamiento del problema, de las interrogantes básicas y del propósito de la investigación.

\section{HIPÓTESIS GENERAL:}

Un programa de capacitación empresarial se relaciona significativamente con el desempeño laboral de los trabajadores del FEBAN.

\section{HIPÓTESIS ESPECÍFICAS:}

1.- Un programa de capacitación empresarial dirigido al Grupo A se relaciona con el desempeño laboral del Grupo Control de los trabajadores del FEBAN.

2.- Un programa de capacitación empresarial dirigido al Grupo B se relaciona con el desempeño laboral del Grupo Control de los trabajadores del FEBAN.

3.- Un Programa de capacitación empresarial dirigido al Grupo C se relaciona con el desempeño laboral del Grupo Control de los trabajadores del FEBAN.

\section{ALCANCES Y LIMITACIONES DE LA INVESTIGACIÓN}

El estudio comprende las diversas sedes del FEBAN a nivel nacional, el cual tiene presencia en todo el Perú, la selección de la muestra ha sido aleatoria, sin remplazamiento y ha permitido tener una representatividad real de los trabajadores del FEBAN.

En el ámbito geográfico al FEBAN, en el período 2006-2007, en cuanto a la revisión bibliográfica, a las fuentes documentales procedentes de bibliotecas y hemerotecas de Lima y provincias, en cuanto al patrocinio económico, este corrió por cuenta del investigador.

\section{IDENTIFICACIÓN Y CLASIFICACIÓN DE VARIABLES IDENTIFICACIÓN DE VARIABLES}

Por la función que cumplen en la hipótesis, las variables macro empleadas en la investigación son:

\section{VARIABLE INDEPENDIENTE}

Programa de capacitación empresarial

\section{VARIABLE DEPENDIENTE}

Desempeño laboral

\section{CLASIFICACIÓN DE VARIABLES}

La clasificación de variables se ha hecho atendiendo a los criterios presentados por el Dr. Elías Mejía Mejía en su Obra "Metodología de la Investigación Científica":

\begin{tabular}{|c|l|l|l|}
\hline $\mathbf{N}^{\circ}$ & Criterio & Denominación & Observación \\
\hline $\mathbf{1}$ & Función en la hipótesis & $\begin{array}{l}\text { Programa de capacitación empresarial: } \\
\text { Variable independiente }\end{array}$ & Causa \\
\hline $\mathbf{2}$ & Naturaleza & Activa, & Manipulable \\
\hline $\mathbf{3}$ & Método de estudio & Cualitativa & \\
\hline $\mathbf{4}$ & Característica & Categórica & Si tiene la característica. \\
\hline $\mathbf{5}$ & Escala & Dicotómica & 0: No hay PCE, 1:PCE \\
\hline
\end{tabular}

\begin{tabular}{|c|l|l|l|}
\hline $\mathbf{N}^{\circ}$ & \multicolumn{1}{|c|}{ Criterio } & \multicolumn{1}{|c|}{ Denominación } & \multicolumn{1}{c|}{ Observación } \\
\hline $\mathbf{1}$ & Función en la hipótesis & $\begin{array}{l}\text { Desempeño laboral: } \\
\text { Variable Dependiente }\end{array}$ & Efecto \\
\hline $\mathbf{2}$ & Naturaleza & Activa, & No manipulable, contrastable \\
\hline $\mathbf{3}$ & Método de estudio & Cualitativa & \\
\hline $\mathbf{4}$ & Característica & Categórica & Si tiene la característica. \\
\hline $\mathbf{5}$ & Escala & Dicotómica & $\begin{array}{l}\text { 0: Bajo desempeño laboral, } \\
\text { 1:Alto desempeño. }\end{array}$ \\
\hline
\end{tabular}




\section{METODOLOGÍA DE LA INVESTIGACIÓN}

En el presente capítulo se especifican los aspectos relacionados con la metodología que se emplea en la investigación y comprende: tipo y nivel de investigación, diseño de la investigación, operacionalizacion de variables, estrategias para la prueba de hipótesis, población y muestra de investigación, técnicas e instrumentos de recolección de información (cuestionario, validación y confiabilidad).

\section{TIPO Y NIVEL DE INVESTIGACIÓN.}

La investigación está orientada a determinar cómo se relaciona la capacitación empresarial con el desempeño laboral de los trabajadores en el FEBAN, Lima. En tal sentido, se puede señalar que en el presente documento, inicialmente se parte de una investigación básica porque proporciona una primera aproximación al problema, limitándose netamente a la descripción del problema. En un segundo lugar, la investigación es de tipo aplicado, pues se realiza un diseño experimental que requiere, para su interpretación, ser explicativo - correlacional,

\section{DISEÑO DE LA INVESTIGACIÓN.}

El diseño de la investigación es experimental que según Hernández, Roberto y otros (2006 p. 205) la define "como la investigación que se realiza mediante la manipulación deliberada de variables. Es decir, la investigación ha tratado de analizar variables que existen en forma natural debido a que las variables independientes (VI) pueden ser manipuladas y controladas por el investigador para ver su efecto sobre otras variables." Al existir la manipulación de variables, se consideró realizar la selección de los sujetos mediante grupos intactos (Hernández, Roberto y otros , 2006 p. 205).

El diseño de la investigación fue experimental, diseño de cuatro grupos aleatorizados con pre prueba y post prueba, de corte transversal, tal como lo define Hernández, Roberto y otros (2006 p.208) caracterizados porque se recolectan datos en un solo momento, en un tiempo único. Su propósito es lograr describir variables y analizar su incidencia o interrelación en un momento dado, mediante la prueba t o mediante pruebas de correlación"

Este tipo de diseño experimental, de corte transversal, comprende modelos estadísticos que explican y describen mediante gráficas o índices aspectos descriptivos, correlacionales y causales que existen entre las variables en un momento determinado.

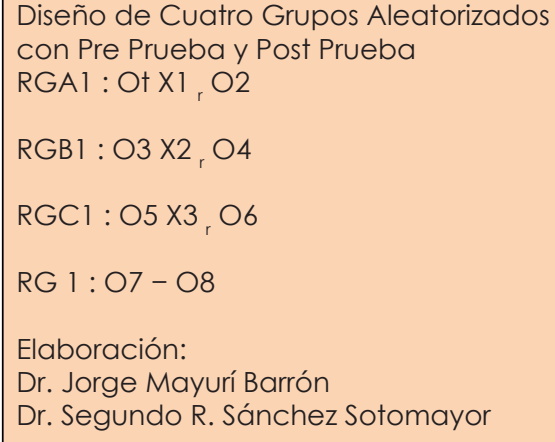

En donde:

$\mathrm{O}$

$\mathrm{X}$

: Es la encuesta aplicada a la población muestral.

: Variable Independiente, Estímulo = Capacitación Empresarial.

Y : Variable Dependiente, Desempeño Laboral

02,04,06, 08 : Se evalúa en un mismo momento

$\mathrm{N} \quad$ : Tamaño de la población.

$r \quad$ : Relación entre las variables en estudio.

\section{ESTRATEGIA PARA LA PRUEBA DE HIPÓTESIS.}

Para probar las hipótesis formuladas se construyeron puntuaciones medias y medianas por cada dimensión de la variable capacitación empresarial, así como para la variable desempeño laboral para luego calcular los coeficientes de la correlación de Pearson y someterlas a la prueba de hipótesis correspondiente mediante el estadístico t- student;

El cálculo del coeficiente de correlación de Pearson, junto al estadístico de prueba t-student, es incorporado en todos los software, particularmente en el programa informático StatisticalPackagefor Social Sciences (SPSS) versión 12.0 en español, la cual se usa en este trabajo de investigación.

El diseño elaborado de cuatro grupos aleatorizados, con pre prueba y post prueba, es una adaptación del tesista del diseño de dos grupos aleatorizados con pre- prueba. En nuestro diseño se toman cuatro grupos intactos, de 20 trabajadores, elegidos de manera aleatoria, y sólo tres de ellos se someten al tratamiento experimental. La finalidad es la de establecer comparaciones 
entre los resultados 02,04,06, 08 de los Grupos sometidos al experimento con el de Control para cuantificar la diferencia entre ellos y sus niveles de causalidad para arribar a las conclusiones terminales de la investigación.

Consideramos que las claves del éxito en nuestro diseño, han sido la ventaja de emplear una segunda prueba al Grupo Control para eliminar el efecto de interacción entre la pre prueba y la manipulación experimental; y el otro hecho el de seleccionar de manera homogénea a los cuatro grupos, de tal forma que cualquiera de ellos pudo recibir el tratamiento.

De tal forma que los resultados para cada caso tanto en el Grupo Control como en el Experimental, nos permite un mejor control estadístico de X (Capacitación empresarial), así como la descripción utilizando medias, barras, gráficas de caja como representaciones visuales de la diferencia de promedios de los grupos control y experimental; y la de contrastar las hipótesis, utilizando el método del valor $\mathrm{p}$ utilizando $\alpha=0.05$, mediante las pruebas de correlación simple, las pruebas t de diferencia de medias, las pruebas del análisis de varianza y la utilización de las gráficas de control de medias y de rangos, para determinar de manera cuantitativa los resultados de la investigación.

\section{OPERACIONALIZACIÓN DE LAS VARIABLES}

En la investigación se ha considerado dos variables: La variable independiente que la conforma la Capacitación Empresarial y la variable dependiente que está conformada por el Desempeño Laboral del personal del FEBAN.

\begin{tabular}{|l|l|l|}
\hline VARIABLE & DIMENSIONES & INDICADORES \\
\hline $\begin{array}{l}\text { Capacita- } \\
\text { ción Em- } \\
\text { presarial }\end{array}$ & Estructura & Pescripciones de cargos \\
& Políticas, reglas y procedimientos \\
& $\begin{array}{l}\text { Responsabi- } \\
\text { lidad }\end{array}$ & $\begin{array}{l}\text { Línea de mando. } \\
\text { Actividades delegadas. } \\
\text { Actividades realizadas. }\end{array}$ \\
\hline & Calor humano \\
\hline & $\begin{array}{l}\text { Delarional. } \\
\text { Relaciones interpersonales de trabajo } \\
\text { Nivel afectivo }\end{array}$ \\
\hline
\end{tabular}

\begin{tabular}{|l|l|l|}
\hline Soporte & $\begin{array}{l}\text { Comunicación } \\
\text { Tipo de liderazgo } \\
\text { Calidad de las interacciones } \\
\text { Equipos de trabajo }\end{array}$ \\
\hline & Recompensa & $\begin{array}{l}\text { Políticas de promoción y ascensos } \\
\text { Reconocimiento }\end{array}$ \\
\hline & Conflicto & $\begin{array}{l}\text { Problemas } \\
\text { Sugerencias } \\
\text { Autocontrol } \\
\text { Actitud }\end{array}$ \\
\hline
\end{tabular}

Variable: Capacitación Empresarial.

Definición Conceptual: Capacitación es toda actividad realizada en una organización, respondiendo a sus necesidades, que busca mejorar la actitud, conocimiento, habilidades o conductas de su personal.

\begin{tabular}{|l|l|l|}
\hline VARIABLE & DIMENSIONES & INDICADORES \\
\hline $\begin{array}{l}\text { Desempe- } \\
\text { ño laboral }\end{array}$ & $\begin{array}{l}\text { Contenido del } \\
\text { trabajo }\end{array}$ & $\begin{array}{l}\text { Desempeño } \\
\text { Responsabilidad } \\
\text { Logro } \\
\text { Estatus } \\
\text { Autonomía }\end{array}$ \\
\hline & Supervisión & $\begin{array}{l}\text { Confianza } \\
\text { Liderazgo } \\
\text { Comunicación }\end{array}$ \\
\hline & $\begin{array}{l}\text { Remuneración } \\
\text { des de ascen- } \\
\text { sos }\end{array}$ & $\begin{array}{l}\text { Sueldos } \\
\text { Prestaciones } \\
\text { Estabilidad laboral }\end{array}$ \\
\hline $\begin{array}{l}\text { Compañeros } \\
\text { de trabajo }\end{array}$ & $\begin{array}{l}\text { Peclasificación } \\
\text { Traslado }\end{array}$ \\
\hline $\begin{array}{l}\text { Cooperación } \\
\text { Confianza }\end{array}$ \\
\hline
\end{tabular}

Variable: Desempeño Laboral.

Definición conceptual: El desempeño laboral es el resultado productivo de una jornada de trabajo.

\section{POBLACIÓN Y MUESTRA.}

La población de estudio en la investigación es el universo del personal de la FEBAN (100 trabajadores a plazo indeterminado) ya que éstos participan de manera exclusiva y cotidiana en las actividades diarias y viven con intensidad las dimensiones que se pretende medir.

Según Salkind (1998). "Una población es un grupo de posibles participantes al cual usted desea generalizar los resultados del estudio" (p. 96); De esta manera y según el cuadro se observa que la población objeto de estudio es conocida 
ya que el FEBAN no cuenta con una población grande y a la vez estratificada; el tamaño global de la muestra se determina por conveniencia en 80 participantes capacitados en planeamiento estratégico, no se consideraron 20 trabajadores de los 100 en total, por ser personal de limpieza y mantenimiento

\section{FIGURA 1: SELECCIÓN DE LA MUESTRA}

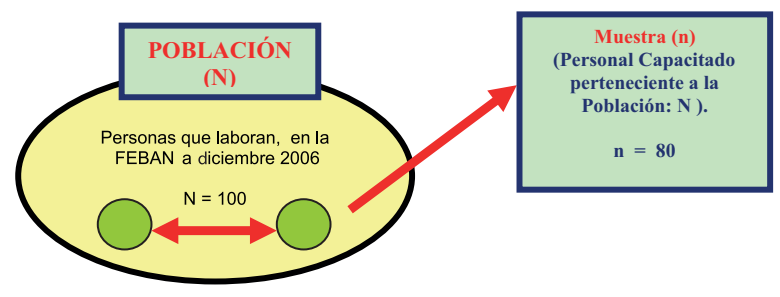

TÉCNICAS E INSTRUMENTOS DE RECOLECCIÓN DE DATOS

Según Hernández, Roberto y otros (2006 p. 274) "recolectar los datos implica elaborar un plan detallado de procedimientos que nos conduzcan a reunir datos con un propósito específico".

Para la recolección de datos se aplicará la observación indirecta a la población muestral por medio de la aplicación de las encuestas al personal de FEBAN, así como a expertos en el área del clima organizacional, del desempeño laboral, de los sicólogos para explicar la interacción de los factores motivacionales. Este instrumento según Quillet, Aristides (1970) lo define como "medio de prueba pre constituido, fundado en el testimonio y sometido por la ley a una serie de formalidades indispensables para su validez y eficacia. (pág. 192) ", y tendrá como insumos para la elaboración del instrumento la operacionalización de la variable.

La Encuesta utilizó en parte la escala de Likert, que consiste en una serie de ítems presentados en forma de afirmaciones o de juicios ante los cuales se pide la reacción de los sujetos (Hernández et al., 200Bn este caso, se empleó un instrumento conteniendo una cantidad de preguntas cerradas específicas para recabar datos relacionados con el desempeño laboral y la segunda parte sobre la capacitación empresarial.

El cuestionario sobre desempeño laboral es un instrumento autoadministrado, con el cual se realizó la medición de la variable a través de los componentes del índice laboral descriptivo: contenido del trabajo, supervisión, remuneración, oportunidades de ascenso y relaciones con los compañeros de trabajo. Incluyó cuatro preguntas, con cuatro alternativas de respuesta: muy insatisfecho, insatisfecho, satisfecho y muy satisfecho

A cada una de las cuatro alternativas que se utilizó en el instrumento, se le asignó un valor numérico que permitió cuantificar las diferentes alternativas de la escala de Likert, que permitiera generar un equivalente numérico y cuantificable respecto a un elemento cualitativo. La escala equivalente se definió como se presenta a continuación (ver Cuadro 1).

Cuadro 1: ESCALA EQUIVALENTE PARA CUANTIFICACIÓN DE VALORES DE ÍTEMS EN EL INSTRUMENTO DE DESEMPEÑO LABORAL

\begin{tabular}{|l|c|c|c|c|}
\hline $\begin{array}{c}\text { Escala } \\
\text { Cualitativa }\end{array}$ & $\begin{array}{c}\text { Muy insa- } \\
\text { tisfecho }\end{array}$ & Insatisfecho & Satisfecho & $\begin{array}{c}\text { Muy satis- } \\
\text { fecho }\end{array}$ \\
\hline $\begin{array}{l}\text { Equi- } \\
\text { valente } \\
\text { numérico }\end{array}$ & 1 & 2 & 3 & 4 \\
\hline
\end{tabular}

El instrumento que midió la variable capacitación empresarial contiene seis preguntas con alternativas múltiples (Ver anexo 2). A cada una de las preguntas se colocó alternativas de respuesta, a las que también se le asignó un valor numérico para poder cuantificarlas, mediante la generación de un equivalente numérico y cuantificable con relación a un elemento cualitativo.

\section{VALIDEZ Y CONFIABILIDAD DEL INSTRUMENTO}

La validez de un instrumento de recolección de datos se refiere al grado en el cual mide, realmente, la variable que se intenta medir, con la participación de varios expertos. Validar un instrumento significa probar que este es veraz, exacto, auténtico, genuino y sólido (Salkind, 1999).

Para la validación de contenido del instrumento sobre capacitación empresarial y desempeño laboral se contó con la información contenida en la operacionalización de variables y dimensiones, en las que se señalaron los indicadores que fueron presentados para su evaluación y fueron aprobados por diez expertos, alcanzando un coeficiente de $64.2 \%$. Se muestran los resultados alcanzados mediante la aplicación del software SPSS Versión 12, de la prueba de Correlación de Friedman. para el instrumento elaborado hallando que el valor $\mathrm{p}=0.00<0.05$, que indica una buena correlación de las preguntas seleccionadas incluidas en nuestra encuesta. 


\begin{tabular}{|ll|l|l|l|l|l|}
\hline & & $\begin{array}{l}\text { Suma de Cuadra- } \\
\text { dos }\end{array}$ & $\begin{array}{l}\text { Grados de li- } \\
\text { bertad }\end{array}$ & Cuadrado Medio & $\begin{array}{l}\text { Friedman Chi Cua- } \\
\text { drado }\end{array}$ & Valor $\mathrm{p}$ \\
\hline Entre Grupos & & 166.035 & 49 & 3.388 & & \\
Inter personal & Entre Items & $418.378(\mathrm{a})$ & 11 & 38.034 & 214.436 & .000 \\
& Residual & 654.705 & 539 & 1.215 & & \\
& Total & 1073.083 & 550 & 1.951 & & \\
Total & 1239.118 & 599 & 2.069 & & \\
\hline
\end{tabular}

Test de Friedman(b): Ítems Estadísticos

\begin{tabular}{|c|c|c|c|}
\hline & Media & $\begin{array}{l}\text { Desviación Es- } \\
\text { tándar }\end{array}$ & $\mathrm{N}$ \\
\hline 1. ¿Qué aspectos requiere su equipo de trabajo para mejorar su desempeño?: & 3.14 & 1.125 & 50 \\
\hline 2. ¿La capacitación puede mejorar el desempeño de las personas en la empresa? & 1.62 & .923 & 50 \\
\hline 3. Su experiencia en cursos de capacitación ha sido: & 2.48 & 1.074 & 50 \\
\hline $\begin{array}{l}\text { 4. Señale ámbitos en los que considere prioritaria la capacitación de su equipo de } \\
\text { Trabajo }\end{array}$ & 4.00 & 2.259 & 50 \\
\hline 5. Señale las causas que originan problemas en su equipo de trabajo: & 2.58 & 928 & 50 \\
\hline 6. En qué áreas requiere usted capacitarse: & 2.60 & 1.738 & 50 \\
\hline 7. ¿Cómo considera usted debe evaluarse el aprovechamiento de la capacitación? & 2.26 & 1.639 & 50 \\
\hline $\begin{array}{l}\text { 8. Los problemas de la empresa pasan porque no se cuenta con una estructura } \\
\text { adecuada de sueldos y salarios: }\end{array}$ & 1.38 & 635 & 50 \\
\hline $\begin{array}{l}\text { 9. ¿Si el curso que se ofrece a su grupo de trabajo es plan Estratégico y desempeño } \\
\text { laboral, lo aceptaría?: }\end{array}$ & 1.30 & .614 & 50 \\
\hline 10. Género & 1.18 & .388 & 50 \\
\hline 11. Profesión & 2.14 & .064 & 50 \\
\hline 12. ¿Si el Curso a dictarse sería liderazgo y desempeño laboral, lo aceptaría?: & 1.70 & .707 & 50 \\
\hline
\end{tabular}

Con respecto a la confiabilidad del instrumento de medición que según Hernández (2006) en su libro clima organizacional se refiere: "al grado en el cual su aplicación repetida a los mismos sujetos produce resultados iguales."

Se aplicó una prueba de confiabilidad del registro, para la cual se utilizo la opinión de los tres (03) mismos expertos, quienes respondieron el cuestionario.

La confiabilidad se realizó para la estructura propia de las preguntas o reactivos. Los valores que arrojó este coeficiente en función del nivel de correlación se categorizó de la siguiente manera:

\begin{tabular}{|l|l|}
\hline Grado de correlación & Confiabilidad \\
\hline 81 a 100 & Excelente correlación \\
\hline 61 a 80 & Buena correlación \\
\hline 41 a 60 & Regular correlación \\
\hline 21 a 40 & Mala correlación \\
\hline 0 a 20 & Pésima correlación \\
\hline
\end{tabular}

Los resultados de la confiabilidad de los instrumentos alcanzó un nivel de buena correlación de $64.2 \%$. 


\begin{tabular}{|l|l|l|l|l|}
\hline Instrumento & $\begin{array}{l}\text { Coeficiente } \\
\text { Alfa de Cronbach's }\end{array}$ & $\begin{array}{l}\text { Alfa de Cronbach's basado } \\
\text { en Items Estandarizados }\end{array}$ & No de Items & Confiabilidad \\
\hline $\begin{array}{l}\text { Capacitación empresarial y desem- } \\
\text { peño laboral }\end{array}$ & .642 & .628 & 12 & Buena correlación \\
\hline
\end{tabular}

Con respecto a la confiabilidad del instrumento de medición para los grupos control y experimental, se aplicó una prueba de confiabilidad utilizándose la opinión de los expertos alcanzando un nivel de excelente correlación de $86.2 \%$

\section{Cuadro No 3: ANÁLISIS DE CONFIABILIDAD}

\begin{tabular}{|c|c|c|c|c|}
\hline Instrumento & $\begin{array}{c}\text { Coeficiente } \\
\text { Alfa de Cronbach's }\end{array}$ & $\begin{array}{c}\text { Alf de Cronbach's basado en Ítems } \\
\text { Estandarizados }\end{array}$ & No de Items & Confiabilidad \\
\hline $\begin{array}{l}\text { Pre Test y } \\
\text { Post Test }\end{array}$ & .862 & .872 & 8 & $\begin{array}{c}\text { Excelente } \\
\text { Correlación }\end{array}$ \\
\hline
\end{tabular}

PLAN DE ANÁLISIS DE RECOLECCIÓN DE RESULTADOS.

La recolección de información se ha realizado orientada por los siguientes lineamientos:

Elaboración de los instrumentos de recolección de datos: Encuestas

Aplicación de los instrumentos: La encuesta se ha aplicado al personal de FEBAN integrante de la población muestral

\section{RESULTADOS}

Para el procesamiento y análisis de la información recolectada se ha utilizado las siguientes técnicas:

En base a la información proporcionada se ha construido las puntuaciones medias y medianas a fin de poder calcular el gráfico de cajas y el coeficiente de correlación de Pearson .

Los resultados hallados son sometido a la prueba de hipótesis por el estadístico t-student, diferencia de medias a fin de aceptar ó rechazar la existencia de una relación entre las variables. Para una mejor explicación del grado de significación se ha utilizado el análisis de varianza y el control estadístico de calidad mediante el uso de las cartas $\mathrm{X}$ y las cartas $\mathrm{R}$.

\section{DISCUCIÓN}

- Como el valor $\mathrm{p}=0.000<0.05$, podemos afirmar con un 95\% que un Programa de capacitación empresarial se relaciona significativamente con el desempeño laboral de los trabajadores del FEBAN.

- Como el valor $\mathrm{p}=0.000<0.05$, podemos afirmar con un $95 \%$ que un programa de capacitación empresarial dirigido al Grupo A se relaciona con el desempeño laboral del Grupo Control de los trabajadores del FEBAN.

- Como el valor $\mathrm{p}=0.000<0.05$, podemos afirmar con un 95\% UnPrograma de Capacitación Empresarial dirigido al Grupo B se relaciona con el Desempeño Laboral del Grupo Control de los Trabajadores del Fondo de Empleados del Banco de la Nación.

- Como el valor $p=0.000<0.05$, podemos afirmar con un $95 \%$ que un programa de capacitación empresarial dirigido al Grupo $\mathrm{C}$ se relaciona con el desempeño laboral del Grupo Control de los trabajadores del FEBAN.

- Como resultado del trabajo de investigación se ha demostrado con el diseño de 4 grupos que los cursos de capacitación mejoran el desempeño laboral de los participantes de manera significativa.

- Los participantes del curso de capacitación consideran que una mejor medición de los resultados de esta capacitación es el promedio de notas final que releja el conocimiento y practica de los conocimientos alcanzados. 


\section{CONCLUSIONES}

Del estudio podemos nosotros considerar a propuesta de los resultados que la mejor evaluación de un curso de capacitación es el promedio de notas, en los cuales se le debería adicionar un trabajo de investigación aplicando estos conocimientos a la realidad de la empresa.

\section{LITERATURA CITADA}

\section{Referida al tema}

1. ACOSTA CONTRERAS, Manual.: Creatividad, Motivación y Rendimiento Académico. Ediciones Aljibe. Málaga. 1998.

2. ARAQUE PERICO, Julian y RIVERA CASTAÑEDA, Nicomedes.. Psicología Organizacional e Industrial. Ediciones Ecoe. Bogotá. 2005.

3. ARISTA M. L. GILDOMERO, CÓRDOVA CADILLO, Alberto.: Psicología de la Motivación. Lima 1974.

4. BEDOYA OROZCO, Mario.: Motivación, Trabajo y Éxito. Colección Humanista. Edt. Venezolana. C.A. Mérida. 1997.

5. COFER C.N., APPLEY M.H.: Psicología de la Motivación. Edit. Profesionales. México. 1987.

6. GONZÁLES FERNÁNDEZ, Antonio.: Motivación Académica. Ediciones Pirámide. Madrid. 2005.

7. GONZÁLEZ LÓPEZ, Luis.: Satisfacción y Motivación en el Trabajo. Ediciones Díaz de Santos. Madrid.2001.

8. GINZBERG, Eli.: Tecnología y Cambio Social. Edit. Hispano-Americana. Ed. 1… México. 1964.

9. HANCOCK, P.: Baudrillard and The Metaphysics of Motivation: a reppraisal of corporate culturalism in the ligt of the work and ideas of Jean Baudrillard. Journal Of Management Studies. Vol. 36 pp.2 1999.

10. IÁKOBSON, Pável M.: La Motivación de la Conducta Humana. Ed. Pueblos Unidos. Montevideo. 1972.

11. MANKELIUNAS, Mateo, V.: Psicología de la Motivación. Edit. Trillas. México. 1987.

12. MAILLO Adolfo.: Enciclopedia de Didáctica Aplicada. Edit. Labor. Barcelona. 1976. T.I.
13. MASLOW A. H.: Motivación y Personalidad. Ediciones Sagitario. Barcelona. 1959.

14. MASLOW Abraham.: El Hombre Autorrealizado. Edit. Kairós. Ed. 15a. Barcelona. 2003.

15. NUTTIN, Joseph, Otros.: Motivación, Emoción y Personalidad. Edit. Paidos. Ed. 2a . Buenos Aires. 1979.

16. NUTTIN, Joseph.: Estructura de la Personalidad. Edit.Kapeluz. Buenos Aires.1968.

17. POLLACK Philip.: Carreras y Oportunidades del Mundo Actual. Edit. Compañía General Fabril. Buenos Aires. 1961.

18. PORTER, Lyman y Steve Lawler: "Teorías de Motivación” Edit Prentice - México 1988

19. REEVE, Johnmarshall. Motivación y Emoción. Edit. McGraw-Hill. Madrid. 1994.

20. SCHUNK Dale H. Teorías del Aprendizaje. Ed. 2a . Edit. Prentice-Hall Hispanoamericana. México. 1997

21. TORO ÁlVAREZ, Fernando.: Desempeño y Productividad. Cincel Ltda. Medellín. 2002.

22. YOUNG, Paúl Thomas.: La Emoción en el Hombre y en el Animal. Buenos Aires. Nova. 1946.

\section{Referida a la Metodología de la Investigación}

1. BROWN Frederick G. Principios de la Medición en Psicología y Educación. Edit. El Manuel Moderno. México. 1980.

2. HAYMAN JOHN L.: Investigación y Educación. Edit. Paidos. Buenos Aires. 1969.

3. HERNÁNDEZ SAMPIERI, Roberto.: Metodología de la Investigación. Ed. 3ạ. Edit. McGrawHill/Interamericana. México. 2006

4. KERLINGER Fred N.: Investigación del Comportamiento. Ed. 2ª . Edit. McGraw-Hill. México, 2002

5. PISCOYA HERMOZA, Luis.: Investigación Científica y Educacional. Ed.1ㄹ. Edit. Amaru. Lima. 1987.

\section{Fuentes Electrónicas}

Cruz Cordero, Teresa. El diagnóstico de la Cultura Organizacional. La experiencia en una Empresa.

http://www. Gestiopolis.com/canales5/ger/ diaorgaex.htm 
R.E.M.E.: Motivación: Conducta y Proceso. (Revista Electrónica de Motivación y Emoción). 1997 www.risolidaria.org.pe.: Lineamientos de Política Educativa 2001 - 2006

http://www.seguridad-la.com/artic/7011.htm

http:/www.azc.uam.mx/publicaciones/gestion/ num11y1/doc14.htm

http/ilo.org/public/spanish/region/ampro/cinferor/publ/labarca/pdf/silveir.pdf

http:/udlap.mx/uddlaconsultores/Default.aspx

http://monografias.com/trabajos34/motivacion-laboral/motivacion-laboral.shtml 